\title{
Actividad anticoagulante in vitro del extracto etanólico de las hojas de dos especies de la familia Euphorbiaceae
}

\section{In vitro anticoagulant activity of the ethanolic extract of the leaves of two species of the family Euphorbiaceae}

\author{
Jhon Ruiz-Baquero'; Oscar Camacho-Romero ${ }^{2}$; Samir Bolívar-González ${ }^{3}$; Álvaro Castro-Zafra ${ }^{4}$
}

\begin{abstract}
'Químico Farmacéutico. Universidad del Atlántico, Facultad de Química y Farmacia, Semillero de Investigación Grupo de Investigación Fitoquímica. Barranquilla Atlántico, Colombia; e-mail: jhonruiz-11a@hotmail.com; (iD) https://orcid.org/0000-0001-7096-6001

${ }^{2}$ Químico Farmacéutico, M.Sc. Universidad del Atlántico, Facultad de Química y Farmacia, Grupo de Investigación Fitoquímica. Barranquilla - Atlántico, Colombia; e-mail: oscarcamacho@mail.uniatlantico.edu.co; iD https://orcid.org/0000-0002-3760-9827

${ }^{3}$ Químico Farmacéutico, Ph.D. Universidad del Atlántico, Facultad de Química y Farmacia, Grupo de Investigación Farmacia Asistencial y Farmacología. Barranquilla - Atlántico, Colombia; e-mail: samirbolivar@mail.uniatlantico.edu.co; iD https://orcid.org/0000-0002-2419-7456

${ }^{4}$ Químico Farmacéutico. Universidad del Atlántico, Facultad de Química y Farmacia, Semillero de Investigación Grupo de Investigación Administración \& Gestión Farmacéutica. Barranquilla - Atlántico, Colombia; e-mail: ajuniorcastro@mail.uniatlantico.edu.co; (iD https://orcid.org/0000-0002-0651-9554
\end{abstract}

Cómo citar: Ruiz-Baquero, J.; Camacho-Romero, O.; Bolívar-González, S.; Castro-Zafra, Á. 2021. Actividad anticoagulante in vitro del extracto etanólico de las hojas de dos especies de la familia Euphorbiaceae. Rev. U.D.C.A Act. \& Div. Cient. 24(2):e1681. http://doi. org/10.31910/rudca.v24.n2.2021.1681

Artículo de acceso abierto publicado por Revista U.D.C.A Actualidad \& Divulgación Científica, bajo una Licencia Creative Commons CC BY-NC 4.0

Publicación oficial de la Universidad de Ciencias Aplicadas y Ambientales U.D.C.A, Institución de Educación Superior Acreditada de Alta Calidad por el Ministerio de Educación Nacional.

Recibido: agosto 16 de 2020

Aceptado: octubre 13 de 2021

Editado por: Helber Adrián Arévalo Maldonado

\section{RESUMEN}

Las enfermedades cardiovasculares (ECV) son un conjunto de trastornos del corazón y de los vasos sanguíneos, que constituyen la principal causa de mortalidad en el mundo. En la búsqueda de alternativas para esta problemática, plantas medicinales de la familia Euphorbiaceae, han sido empeladas con fines terapéuticos, para prevenir, atenuar o curar los efectos generados por estas enfermedades. El objetivo de este trabajo fue conocer el perfil fitoquímico y evaluar la actividad anticoagulante in vitro de los extractos etanólicos de las hojas de Croton malambo y Acalypha hispida sobre plasma humano. Para ello, se obtuvo el extracto de las hojas y se le realizó el tamizaje fitoquímico, la evaluación del Tiempo de Tromboplastina Parcial activada (TTPa) y del Tiempo de Protombina (TP). En el perfil fitoquímico, se confirmó la presencia de alcaloides, taninos, flavonoides, leucoantocianidinas, fenoles, sesquiterpenlactonas, glucósidos cardiotónicos y terpenos.
En la actividad anticoagulante, se evidenció la inhibición de la coagulación en la vía intrínseca, obteniendo resultados significativos para el TTPa, a diferencia que el test TP, donde los resultados obtenidos se encontraron similares al control. Esta investigación demuestra la acción anticoagulante de las plantas, ya que induce, significativamente, a una mayor prolongación del tiempo de coagulación; ambas especies presentaron una mayor actividad, a $200 \mathrm{mg} / \mathrm{mL}$.

Palabras clave: Acalypha hispida; Croton malambo; Plantas medicinales; Tiempo de Tromboplastina Parcial activada; Tiempo de Protombina.

\section{ABSTRACT}

Cardiovascular diseases (CVD) are a group of disorders of the heart and blood vessels, which correspond to the principal 
causes of death in the world. In the search for alternatives to this problem, medicinal plants of the Euphorbiaceae family have been investigated for therapeutic purposes to prevent, attenuate or cure the effects generated for these illnesses. The objective of this work was to know the phytochemical profile and evaluate the anticoagulant activity in vitro of the ethanolic extracts of the leaves of Croton malambo and Acalypha bispida on human plasma. For this, extract of their leaves was obtained, and phytochemical screening was performed, as well as the evaluation of the Activated Partial Thromboplastin Time (aPTT) and the Prothrombin Time (TP). The phytochemical profile confirmed the presence of alkaloids, tannins, flavonoids, leucoanthocyanidins, phenols, sesquiterpene lactones, cardiotonic glycosides, and terpenes. In the anticoagulant activity, the inhibition of coagulation in the intrinsic pathway was evidenced, obtaining significant results for aPTT, unlike the TP test where the results obtained were like the control. This research demonstrates the effectiveness of the plant with anticoagulant action since they significantly induce a longer prolongation of the clotting time, both species showed higher activity at $200 \mathrm{mg} / \mathrm{mL}$.

Keywords: Acalypha hispida; Croton malambo; Medicinal plants; Activated Partial Thromboplastin Time; Prothrombin Time.

\section{INTRODUCCIÓN}

Los eventos isquémicos en el miocardio y los accidentes cerebrovasculares suelen ser fenómenos agudos, que se deben, sobre todo, a obstrucciones que impiden que la sangre fluya hacia el corazón o el cerebro (Oberreuter et al. 2014); una de las causas más frecuentes es la formación de coágulos de sangre (trombos) en las venas, que se pueden desprender y alojar en los vasos del corazón y del cerebro (OMS, 2015).

Actualmente, en el mundo, las enfermedades cardiovasculares representan la principal causa de mortalidad. Para 2008, causaron el $63 \%$ de las muertes y se estima un incremento del $15 \%$ (44 millones), entre 2010 y 2020, alcanzando el $73 \%$ de la mortalidad y el $60 \%$ de la carga de la morbilidad (ONS, 2013). En Colombia, entre 2005 y 2017, la principal causa de muerte en la población general fueron las enfermedades del sistema circulatorio, las cuales, presentaron una tendencia decreciente en el tiempo, pasando de 166,43 a 150,30 muertes por cada 100.000 habitantes (Minsalud, 2018).

A nivel clínico, el tratamiento de los trombos se trata con anticoagulantes sintéticos (Jerjes-Sánchez et al. 2017); sin embargo, presentan efectos adversos, como trombocitopenia, urticaria, asma, anafilaxis, entre otras (Warkentin et al. 1995), lo que resulta en una problemática más relacionada con las enfermedades cardiovasculares (Rocha et al. 2004; Trejo I., 2004). En la búsqueda de nuevos fármacos anticoagulantes existe un mayor interés por el uso de plantas medicinales que presenten estas actividades biológicas y disminuyan la presencia de efectos secundarios, teniendo en cuenta que, en la actualidad, se presenta una tendencia progresiva por el uso de los sistemas tradicionales de medicina (Gallegos-Zurita, 2016).
Croton malambo y Acalypha hispida son dos especies de la familia Euphorbiaceae que se estudian por su actividad biológica, como es el caso de su uso como anticoagulante (Morón Rodríguez et al. 2007; Barragán Zarate et al. 2016). El género Croton, se caracteriza porque sus especies poseen una gran cantidad de usos, a nivel etnobotánica; también, se reportan varios usos interesantes en la medicina tradicional y se destaca la presencia de metabolitos secundarios, que se caracterizan porque poseen un amplio rango de actividad biológica (Coy Barrera et al. 2016; Matara et al. 2021). En el caso de $A$. hispida, se caracteriza por sus constituyentes con potencial para el tratamiento de muchas patologías, debido a su actividad antiinflamatoria y antioxidante (Siraj et al. 2016). Varias investigaciones reportan las diferentes actividades biológicas que presentan estas dos especies de Euphorbiaceae, dado que la mayoría de sus aceites esenciales presentan propiedades curativas (Jaramillo C. et al. 2010; Wardoyo et al. 2021).

Con base en lo anterior, se determinó el perfil fitoquímico y la actividad anticoagulante in vitro, a través de las técnicas Tiempo de Tromboplastina Parcial Activada (TTPA) y Tiempo de Protrombina (TP), de los extractos etanólicos de las hojas de C. malambo y $A$. bispida, sobre plasma humano.

\section{MATERIALES Y MÉTODOS}

Este trabajo, se realizó en las instalaciones del Laboratorio de Investigaciones-Productos Naturales adscrito a la Facultad de Química y Farmacia, de la Universidad del Atlántico.

Recolección del material vegetal. La colecta de las hojas de C. malambo y A. hispida, se realizó en el corregimiento de Paluato, Galapa-Atlántico, con coordenada geográficas $10^{\circ} 48^{\prime} \mathrm{N}, 74^{\circ} 54^{\prime} \mathrm{O}$ y Salgar, Puerto Colombia-Atlántico, con coordenada geográficas $11^{\circ} 0^{\prime} \mathrm{N}$ y $74^{\circ} 47^{\prime} \mathrm{O}$, a $18 \mathrm{~m}$ s.n.m. y ambas zonas presentan condiciones similares de temperatura $\sim 30{ }^{\circ} \mathrm{C}$ y humedad relativa $\sim 75 \%$, aprox., a las 7 a.m. Las plantas crecieron en condiciones de campo abierto y las muestras se tomaron de plantas sin evidencia de afectación por fitopatógenos o ataque de insectos. Las especies están situadas en la costa del Mar Caribe, zona con suelo franco arcilloso, sustrato que proporciona condiciones adecuadas para la absorción de los nutrientes. Para la identificación, se tomaron dos muestras de cada especie y se enviaron al Herbario Nacional Colombiano del Instituto de Ciencias Naturales de la Universidad Nacional de Colombia, sede Bogotá.

Obtención de los extractos etanólicos. Se realizó un proceso de limpieza y de secado del material vegetal a temperatura ambiente. Posteriormente, se realizó el proceso de molienda, para obtener un tamaño de partícula moderadamente fino y llevado al método de extracción por maceración (Terán Portelles et al. 2019), con etanol al $96 \%$, durante 8 días, con agitación esporádica. Finalmente, se filtró y se concentró con un rotaevaporador R-10, a $38^{\circ} \mathrm{C}$, hasta obtener un extracto seco (Camacho-Romero et al. 2019).

Tamizaje fitoquímico preliminar. Los extractos fueron sometidos a un screening fitoquímico cualitativo, a través de 
ensayos de coloración y de precipitación (Martínez, 2008), con el fin de confirmar los principales constituyentes químicos presente en los extractos etanólicos, de las hojas de C. malambo y A. hispida. Para el caso de alcaloides, esteroides y triterpenoides, se emplearon las pruebas descritas por Gil-Frómeta et al. (2019) y para taninos, saponinas, leucoantocianidinas, flavonoides, fenoles, antraquinonas y sesquiterpenlactonas, las pruebas mencionadas por Beltrán Villanueva et al. (2013) y Soto Vásquez (2015).

Evaluación de la actividad anticoagulante in vitro. A partir de los extractos secos de C. malambo y A. hispida, se tomaron $5 \mathrm{~g}$ y se aforaron en un matraz de $25 \mathrm{~mL}$, obteniéndose la concentración de $200 \mathrm{mg} / \mathrm{mL}$; de igual modo, se realizaron diluciones de $10,50 \mathrm{y}$ $100 \mathrm{mg} / \mathrm{mL}$. Posteriormente, se realizó la prueba de coagulación, utilizando las técnicas de tiempo de tromboplastina parcial activada (TTPA) y el tiempo de protrombina (TP) y, como control negativo, se utilizó plasma sanguínea normal, es decir, el valor de referencia, en la cual, coagula la sangre en estado normal.
Análisis estadístico. El análisis estadístico, se realizó mediante el uso de las pruebas ANOVA y Tukey, para tamaños de muestras iguales, con un nivel del 95,0 \% de confianza y $\mathrm{p}$ $<0,05$. Para establecer la correlación entre las variables, se consolidó la base de datos en Microsoft Excel y se realizó el plan de análisis con estadística descriptiva, para conocer la capacidad de formación del coágulo, de manera similar en las muestras, donde todas se realizaron por triplicado, a través del programa estadístico Statgraphics Centurion (XV).

\section{RESULTADOS Y DISCUSIÓN}

Perfil fitoquímico. En la tabla 1, se observan los resultados cualitativos obtenidos en la marcha fitoquímica preliminar de los extractos etanólicos de las hojas de C. malambo y $A$. hispida, los cuales, mostraron resultados positivos para metabolitos secundarios, como alcaloides, flavonoides, esteroides y triterpenos, taninos, leucoantocianidinas, sesquiterpenlactonas y fenoles y negativo para los grupos químicos de saponinas y de antraquinonas.

Tabla 1. Marcha fitoquímica preliminar de las hojas de Croton malambo y Acalypha hispida.

\begin{tabular}{|c|c|c|c|}
\hline Tipo de compuesto & Pruebas (reactivos) & C. malambo & A. hispida \\
\hline \multirow{2}{*}{ Alcaloides } & Hager & +++ & +++ \\
\hline & Wagner & +++ & +++ \\
\hline Esteroides y triterpenoides & Lieberman-Burchard & + & +++ \\
\hline \multirow{3}{*}{ Taninos } & Gelatina-Sal & +++ & ++ \\
\hline & Dicromato de potasio & +++ & ++ \\
\hline & Cafeína & + & + \\
\hline Saponinas & Espuma & - & - \\
\hline \multirow[b]{2}{*}{ Leucoantocianidinas } & Rosenhein & ++ & + \\
\hline & $\begin{array}{l}\text { Ácido clorhídrico } \\
\text { concentrado }\end{array}$ & ++ & ++ \\
\hline \multirow{2}{*}{ Flavonoides } & Shinoda & ++ & ++ \\
\hline & Pews & + & +++ \\
\hline $\begin{array}{l}\text { Sesquiterpenlactonas y } \\
\text { glicósidos cardiotónicos }\end{array}$ & Baljet & ++ & ++ \\
\hline Fenoles & Tricloruro férrico & +++ & +++ \\
\hline Antraquinonas & Acetato de magnesio & - & - \\
\hline
\end{tabular}

No hay presencia $(-)$, se encuentra en poca cantidad $(+)$, se encuentra en mediana cantidad $(++)$, se encuentra en gran cantidad $(+++)$.

Actividad anticoagulante. Los extractos de las especies C. malambo y $A$. bispida fueron probados a diferentes concentraciones, para evaluar su actividad anticoagulante y los resultados obtenidos, se presentan en la tabla 2; están expresados en segundos transcurridos hasta la formación del coágulo. Las pruebas empleadas para la evaluación de los tiempos de coagulación fueron: tiempo de tromboplastina parcialmente activa, la cual, su prolongación (tiempo que se demora en formar un coágulo sanguíneo) indica inhibición de la vía intrínseca y tiempo de protrombina, donde su prolongación indica inhibición de la vía extrínseca de la cascada de coagulación (Zamora-González, 2012).

En la tabla 2, se puede observar que, a concentración de $200 \mathrm{mg} / \mathrm{mL}$, el extracto de C. malambo registró un tiempo de coagulación promedio de 39,1 segundos, mientras que el extracto de $A$. bispida, a la misma concentración, presentó un tiempo de coagulación promedio de 41,8 segundos. Según lo anterior, a estas concentraciones, los extractos se catalogan como sustancias 
Tabla 2. Actividad anticoagulante In vitro mediante TTPa y TP de Croton malambo y Acalypha hispida.

\begin{tabular}{|c|c|c|c|c|}
\hline Especie & $\begin{array}{c}\text { Concentraciones } \\
(\mathbf{m g} / \mathbf{m L})\end{array}$ & $\begin{array}{c}\text { TTPa } \\
\text { (segundos)* }\end{array}$ & $\begin{array}{c}\text { TP } \\
\text { (segundos)* }\end{array}$ & $\begin{array}{c}\text { TP } \\
(\text { INR)* }\end{array}$ \\
\hline \multirow{4}{*}{ C. malambo } & 10 & $29,7 \pm 0,06^{\mathrm{a}}$ & $12,2 \pm 0,00^{\mathrm{e}}$ & $1,03 \pm 0,06^{\mathrm{d}}$ \\
\cline { 2 - 5 } & 50 & $34,8 \pm 0,00^{\mathrm{d}}$ & $12,2 \pm 0,00^{\mathrm{e}}$ & $1,03 \pm 0,00^{\mathrm{d}}$ \\
\cline { 2 - 5 } & 100 & $38,1 \pm 0,00^{\mathrm{e}}$ & $12,2 \pm 0,00^{\mathrm{e}}$ & $1,03 \pm 0,00^{\mathrm{d}}$ \\
\hline & 200 & $39,1 \pm 0,00^{\mathrm{g}}$ & $12,6 \pm 0,00^{\mathrm{f}}$ & $1,06 \pm 0,00^{\mathrm{e}}$ \\
\hline \multirow{3}{*}{ A. hispida } & 10 & $32,1 \pm 0,04^{\mathrm{b}}$ & $12,2 \pm 0,05^{\mathrm{e}}$ & $1,03 \pm 0,00^{\mathrm{d}}$ \\
\cline { 2 - 5 } & 50 & $34,1 \pm 0,00^{\mathrm{c}}$ & $11,2 \pm 0,00^{\mathrm{c}}$ & $0,94 \pm 0,00^{\mathrm{b}}$ \\
\cline { 2 - 5 } & 100 & $38,6 \pm 0,00^{\mathrm{f}}$ & $9,6 \pm 0,00^{\mathrm{b}}$ & $0,81 \pm 0,00^{\mathrm{a}}$ \\
\cline { 2 - 5 } & 200 & $41,8 \pm 0,00^{\mathrm{h}}$ & $9,5 \pm 0,00^{\mathrm{a}}$ & $0,80 \pm 0,00^{\mathrm{a}}$ \\
\hline \multicolumn{2}{|c|}{ Control negativo } & $29,7 \pm 0,01^{\mathrm{a}}$ & $11,9 \pm 0,00^{\mathrm{d}}$ & $1,00 \pm 0,00^{\mathrm{c}}$ \\
\hline
\end{tabular}

* Medias con letras dentro de cada variable para mirar grupos en subconjuntos homogéneos, según prueba de Tukey, para $\mathrm{p} \leq 0,05$ ( $\mathrm{n}=3$ ) \pm DS Desviación estándar.

que demoran la formación del coágulo, puesto que sobrepasan los 10 segundos obtenidos con el control negativo; no obstante, en términos generales, las concentraciones de 50 y $10 \mathrm{mg} / \mathrm{mL}$, para ambas especies, mostraron resultados dentro de los rangos normales de 25 a 35 segundos, en comparación con el control negativo, los cuales, contabilizan hasta 6 segundos por encima o por debajo del valor obtenido en el control negativo (ZamoraGonzález, 2012).

En ambas especies, se evidenció una actividad anticoagulante significativa para el TTPa, en comparación con el control negativo 29,7 segundos, registrando tiempos de coagulación más amplios a este, a excepción de C. malambo, a $10 \mathrm{mg} / \mathrm{mL}$, que presentó el mismo valor. Además, se demostró proporcionalidad, corroborada al observar que, en la medida que las concentraciones aumentaron, los tiempos de coagulación se fueron prolongando; por otra parte, el efecto anticoagulante no se evidenció de acuerdo con los resultados obtenidos por la prueba del TP, donde la prueba ANOVA mostró que no había diferencia significativa entre el valor del TP control y las concentraciones de los extractos de C. malambo y A. bispida.

Estudios realizados con el género Croton, en el que se analizaron 14 especies, se determinaron la presencia de metabolitos secundarios, como alcaloides, taninos, fenoles simples, glucósidos cardiotónicos, triterpenos y flavonoides (Payo Hill et al. 2001). En otro estudio realizado con otras especies, como C. mutisianus, C. polycarpus, C. phyllanthus, C. securinega y C. schiedeanus, se notó la ausencia de saponinas y se confirmó la presencia de alcaloides, flavonoides, taninos y terpenos (Payo Hill et al. 2001). En otro estudio, en el que se incluyó a C. malambo, Jaramillo C. et al. (2010) encontraron los metabolitos alcaloides, terpenos, taninos, fenoles, flavonoides, sesquiterpenlactonas y glucósidos cardiotónicos, metabolitos que concuerdan con los hallados en el presente estudio.

Para A. bispida, se reporta la presencia de alcaloides, flavonoides, taninos, glucósidos cardiotónicos y fenoles, y la ausencia de saponinas y de antraquinonas (Onocha et al. 2011; Osarumwense \& Okunrobo, 2013; Manikanta et al. 2014); dichos resultados concuerdan con los obtenidos en el estudio. Estos compuestos demuestran el potencial terapéutico que tienen estas plantas como antiinflamatorio (Siraj et al. 2016), hipoglucémico (Alfarisi et al. 2020) y antibacteriano (Noumedem et al. 2013).

El modelo clásico de la coagulación, descrita por Davie \& Ratnoff (1964), muestra cómo ocurre el proceso enzimático en cascada (Gómez Baute et al. 2011), que se divide por dos vías, la intrínseca y la extrínseca, siendo evaluadas por las pruebas de laboratorio tiempo de tromboplastina parcial activada (TTPA) y tiempo de protrombina (TP), respectivamente. En la vía intrínseca participan los factores intrínsecos de la sangre (factores VIII, IX y XI) y la vía extrínseca, se caracteriza por requerir una sustancia activadora extrínseca, el factor tisular (Palomo et al. 2009).

$\mathrm{Al}$ analizar los resultados obtenidos frente a la evidencia (Figura 1), se aprecia concordancia con los efectos anticoagulantes de otras plantas, como la Ricinus conmunis y Jatropha curcas, que también pertenecen a las euforbiáceas; cabe resaltar que Gallardo-Vásquez et al. (2019) utilizaron para su investigación el látex de J. curcas, lo que podría justificar la mayor prolongación del tiempo de coagulación, 56 segundos, a diferencia del promedio de 40 segundos, de las otras plantas. Además, según la literatura, la Warfarina prolonga el tiempo de coagulación por encima de los 60 segundos, a concentraciones muy bajas; esto quiere decir que, este agente químico de efecto comprobado, presenta mayor eficiencia que los extractos de las especies vegetales estudiadas (Ku \& Bae, 2014).

En los valores de INR (prueba de tiempo de protrombina) para cada prueba del tiempo de tromboplastina, se observó que los resultados de las dos especies se encuentran dentro de los referenciados para esta prueba 0,8-1,2. Gallardo-Vásquez et al. (2019) reportaron en su investigación con el látex de $J$. curcas resultados similares a los encontrados en este estudio, al obtener tiempos de coagulación más prolongados, mediante el test TTPa, pero el TP no presentó resultados significativos con referencia a los controles negativo. Por tanto, los extractos de C. malambo y $A$. hispida inhiben la coagulación en la vía intrínseca, por lo que se espera que actúe sobre alguno de los factores responsables de la conversión de la protombina en trombina (factores VII, IX, XI y XII de la cascada de coagulación); pero no actúan sobre la vía extrínseca. Los medicamentos, como la Heparina y Warfarina, también actúan sobre la vía intrínseca, inhibiendo algunos de estos factores (Trejo I., 2004). 


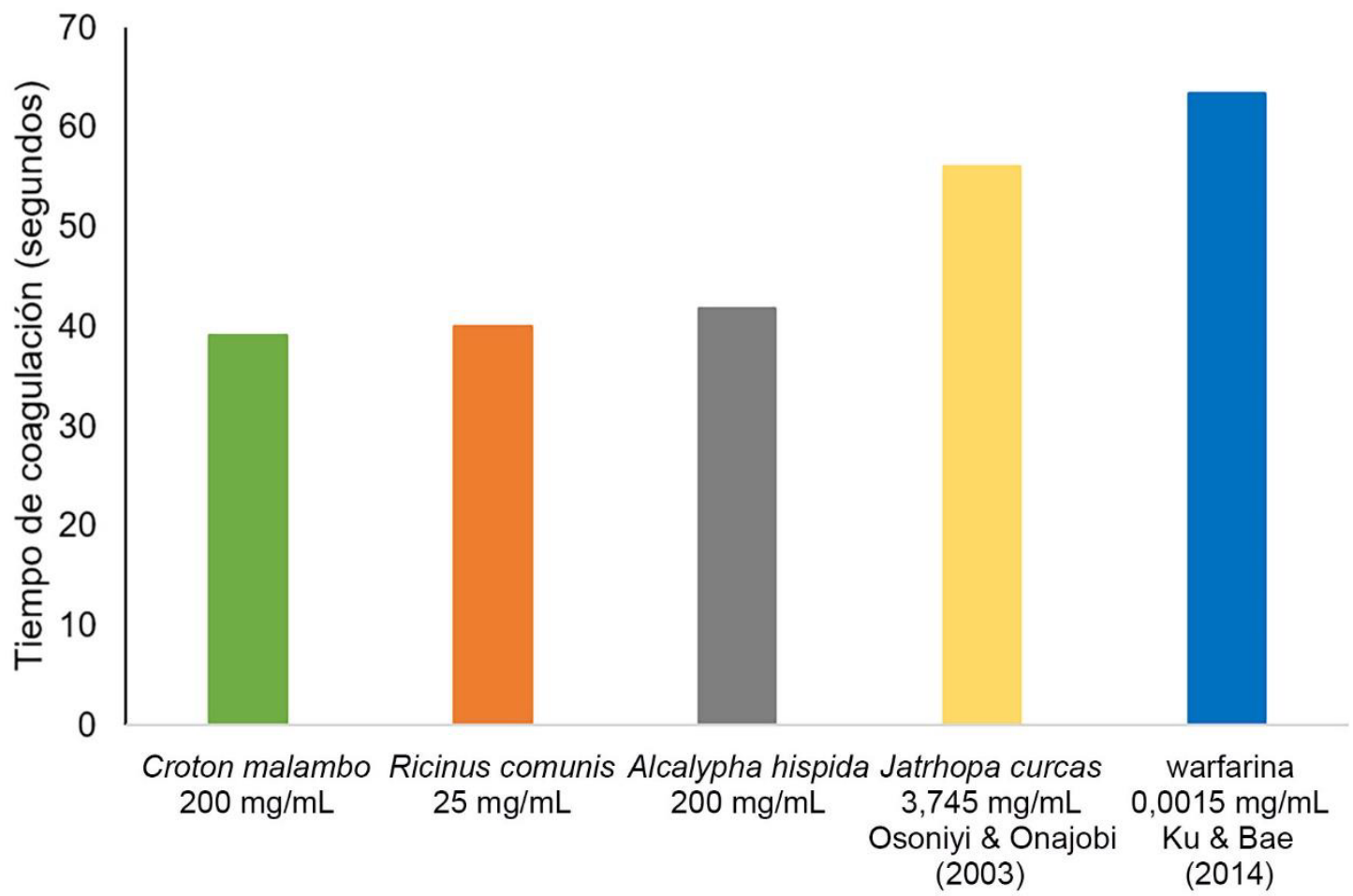

Figura 1. Comparación del tiempo de coagulación obtenido por TTPa reportado en la literatura (Osoniyi \& Onajobi, 2003; Ku \& Bae, 2014).

Se puede concluir que el extracto etanólico de las dos especies evaluadas en dosis de $200 \mathrm{mg} / \mathrm{mL}$ actuó como un anticoagulante, inhibiendo la coagulación en la vía intrínseca, vía por la que actúan los medicamentos anticoagulantes mayormente empleados, como la Heparina y Warfarina, alargando los tiempos de coagulación en las pruebas empleadas en condiciones in vitro.

Conflictos de intereses: Los autores declaran que no existe conflicto de intereses que ponga en riesgo la validez de los resultados presentados.

\section{REFERENCIAS}

1. ALFARISI H.; SA'DIAH S.; WRESDIYATI, T. 2020. Polyphenol profile, antioxidant and hypoglycemic activity of Acalphba bispida leaf extract. Indian Journal of Pharmaceutical Sciences. 2(2):291-299.

2. BARRAGÁN ZARATE, G.S.; LAGUNEZ, L.; SOLANO, R. 2016. Perfil fitoquímico y evaluación de actividad anticoagulante de hojas, flores y pseudobulbos de Prosthechea karwinski. Revista Mexicana de Agroecosistemas. 3(supl. 3):267-275.

3. BELTRÁN VILLANUEVA, C.E.; DÍAZ CASTILLO, F.; GÓMEZ ESTRADA, H. 2013. Tamizaje fitoquímico preliminar de especies de plantas promisorias de la costa atlántica colombiana. Revista Cubana de Plantas Medicinales. 18(4):619-631.
4. CAMACHO-ROMERO, O.I.; BARRIOS-MÁRQUEZ, S.; LOZANO-CONTRERAS，E.; GARCÍA-VILORIA，L. 2019. Actividad larvicida de extractos hidroalcohólicos de Pala scholaris (L.) Roberty sobre larvas de estadio III de Aedes aegypti. Journal of Negative \& No Positive Results. 4(10):1022-1031. https://doi.org/10.19230/jonnpr.3081

5. COY BARRERA, C.A.; CONSTANZA GÓMEZ, D.; CASTIBLANCO, F.A. 2016. Importancia medicinal del género Croton (Euphorbiaceae). Revista Cubana de Plantas Medicinales. 21(2):234-247.

6. DAVIE, E.W.; RATNOFF, O.D. 1964. Waterfall sequence for intrinsic blood clotting. Science. 145:1310-1312. https://doi.org/10.1126/science.145.3638.1310

7. GALLARDO-VÁSQUEZ, G.J.; CHÁVEZ-FLORES, J.E.; CONTRERAS-TORVISCO, M. 2019. Evaluación del efecto antibacteriano del látex de Jatropha curcas "piñón" frente a Staphylococcus aureus. Duazary. 16(1):105-114. https://doi.org/10.21676/2389783X.2533

8. GALLEGOS-ZURITA, M. 2016. Las plantas medicinales: principal alternativa para el cuidado de la salud, en la población rural de Babahoyo, Ecuador. Anales Facultad de Medicina. 77(4):327-332. 
9. GIL-FRÓMETA, S.; ESCALONA-ARRANZ, J.C.; GARCÍADÍAZ, J.; FERNÁNDEZ-HEREDIA, J.A.; MACHADO, R. 2019. Phytochemical, and antimicrobial evaluation of the essential oil and extracts of the leaves of Amyris elemifera $\mathrm{L}$. (yellow coaba). Revista Cubana de Química. 31(3):427-439.

10. GÓMEZ BAUTE, R.A.; GUERRA ALFONSO, T.; DITA SALABERT, L.; FERNÁNDEZ ÁGUILA, J.L.; CABRERA ZAMORA, M. 2011. Teoría celular de la coagulación: de las cascadas a las membranas celulares. MediSur. 9(2):146-155.

11. JARAMILLO C., B.E.; DUARTE, E.; MUÑOZ, K.; STASHENKO, E. 2010. Composición química volátil del aceite esencial de Croton malambo H. Karst. colombiano y determinación de su actividad antioxidante. Revista Cubana de Plantas Medicinales. 15(3):133-142.

12. JERJES-SÁNCHEZ, C.; CANTÚ-BRITO, C.; ARAUZ, A.; MARTÍNEZ-SÁNCHEZ, C.; SANDOVAL-ZÁRATE, J.; RAMÍREZ-RIVERA, A.; ANAYA-SANTACRUZ, E.; ARGÜELLES-MORALES, N.; ARIAS-MENDOZA, A.; ARRIAGA-NAVA, R.; BRISEÑO-DE LA CRUZ, J.L.; CARRILLO-CALVILLO, J.; CASSAGNE, G.; CHÁVEZPÁEZ, J.; CHIQUETE-ANAYA, E.; EID-LIDT, G.; ELIZALDE-GONZÁLEZ, J.J.; ESCAMILLA-GARZA, J.M.; FABIANI, M.A.; FLORES-SILVA, F.D.; GÓMEZ-Y ÁLVAREZ， E.B.; GONZÁLEZ-AZUARA， L.R.; GUTIÉRREZ-FAJARDO, P.; LEÓN-HERNÁNDEZ, F.J.; MÁRQUEZ-ROMERO, J.M.; MURILLO-BONILLA, L.M.; PULIDO-ZAMUDIO, T.; REYES-MÉNDEZ, H.; RUIZ-FRANCOA, A.E.; RUIZ-SANDOVAL, J.L.; SÁNCHEZ-APARICIO, H.E.; SEOANE-GARCÍA DE LEÓN, J.M.; VENEGAS-TORRES, J.A.; VENZORCASTELLANOS, J.P.; VILLARREAL-CAREAGA, J.; ZAYAS-HERNÁNDEZ, N.G. 2017. Guías para Anticoagulación y Trombosis del Tromboembolismo Venoso, Infarto con Elevación del ST, Cardioembolismo Cerebral y del Infarto Cerebral Agudo. Archivos de Cardiología de México. 87(supl 1):1-66.

13. KU, S.K.; BAE, J.S. 2014. Antiplatelet, anticoagulant, and profibrinolytic activities of withaferin A. Vascular Pharmacology. 60(3):120-126.

https://doi.org/10.1016/j.vph.2014.01.009

14. MANIKANTA, B.; VARMA, P.; KRISHNA, P.; KUMAR, A.; RATHINAM, K. 2014. Phytochemical and antidiarrhoeal activity of combined extracts of Acalpha hispida, Acalypha nervosa and Acalypha fruiticosa. Journal of Chemical and Pharmaceutical Sciences. 7(3):194-196.

15. MARTÍNEZ, A. 2008. Manual de prácticas de laboratorio de farmacognosia y fitoquímica 2008. Universidad De Antioquia.
16. MATARA, D.N.; NGUTA, J.M.; MUSILA, F.M.; MAPENAY, I.; ALI, H.M.; OMAMBIA, V.M. 2021. Botanical description, ethnomedicinal uses, phytochemistry and pharmacological effects of Croton dichogamus Pax (Euphorbiaceae). The Journal of Phytopharmacology. 10(1):42-47.

17. MINISTERIO DE SALUD Y PROTECCIÓN SOCIAL, MINSALUD. 2018. Análisis de situación de salud (ASIS). Disponible desde Internet en: https://www.minsalud.gov. $\mathrm{co} /$ sites/rid/Lists/BibliotecaDigital/RIDENS/ED/PSP/a sis-colombia-2018.pdf (con acceso 25/02/2018)

18. MORÓN RODRÍGUEZ, F.J;; GUERRERO JÁCOME, R.O.; VICTORIA AMADOR, M. DEL C. 2007. Plantas medicinales caribeñas con potencialidad para inhibir la agregación de las plaquetas. Revista Cubana de Plantas Medicinales. 12(2).

19. NOUMEDEM, J.; DE DIEU TAMOKOU, J.; NGO TEKE, G.; MOMO, R.; KUETE, V.; KUIATE, J.R. 2013. Phytochemical analysis, antimicrobial and radical-scavenging properties of Acalypha manniana leaves. SpringerPlus. 2:503.

20. OBERREUTER, G.; SILVA, N.; CABA, S.; MORALES, M.; NIETO, E.; GUEVARA, C. 2014. Accidente cerebrovascular isquémico en pacientes con trombo intracavitario: Experiencia con tratamientos distintos en fase aguda. Revista Médica de Chile. 142(9):1200-1204. http://dx.doi.org/10.4067/S0034-98872014000900015

21. OBSERVATORIO NACIONAL DE SALUD, ONS. 2013. Enfermedad cardiovascular principal causa de muerte en Colombia. Boletín técnico Observatorio Nacional de Salud No. 1. Disponible desde Internet en: https://www.ins.gov. co/Direcciones/ONS/Boletines/boletin_web_ONS/ boletin1.html (con acceso 18/04/2020).

22. ONOCHA, P.A.; OLOYEDE, G.K.; AFOLABI, Q.O. 2011. Chemical composition, cytotoxicity and antioxidant activity of essential oils of Acalppha hispida flowers. International Journal of Pharmacology. 7(1):144-148. https://dx.doi.org/10.3923/ijp.2011.144.148

23. ORGANIZACIÓN MUNDIAL DE LA SALUD, OMS. 2015. Enfermedades cardiovasculares. Disponible desde Internet en: http://www.who.int/mediacentre/factsheet s/fs317/es/ (con acceso 18/04/2020).

24. OSARUMWENSE, P.O.; OKUNROBO, L.O. 2013. Phytochemical Screening and the Antimicrobial Properties of Acalypha hispida Burm. F. (Euphorbiaceae). African Journal of Phamaceutical Research \& Development. 5(1):36-39.

25. OSONIYI, O.; ONAJABI, F. 2003. Coagulant and anticoagulant activities in Jatropha curcas latex. Journal of 
Ethnopharmacology. 89 (1):101-105.

https://doi.org/10.1016/s0378-8741(03)00263-0

26. PALOMO, I.; MUÑOZ, B.; RETAMALES, E.; HIDALGO, P.; PANES, O.; ARTIGAS, C.; PEREIRA, J. 2009. Estudio de laboratorio de las enfermedades hemorragiparas. En: Palomo, L.; Pereira, J.; Palma, J. (eds). Hematología, fisiopatología y diagnóstico. Ed. Universidad de Talca. p.310-319.

27. PAYO HILL, A.; DOMINICIS, M.E.; MAYOR,J.;OQUENDO, M.; SARDUY, R. 2001. Tamizaje fitoquímico preliminar de especies del género Croton L. Revista Cubana de Farmacia. 35(3):203-206.

28. ROCHA, H.A.O.; FARIAS, E.H.C.; BEZERRA, L.C.L.M.; ALBUQUERQUE, I.R.L.; MEDEIROS, V.P.; QUEIROZ, K.C.S.; LEITE, E.L. 2004. Polissacarídeos sulfatados de algas marinhas com atividade anticoagulante. InfarmaCiencias Farmacéuticas. 16(1-2):82-87.

29. SIRAJ, M.A.; SHILPI, J.A.; HOSSAIN, M.G.; UDDIN, S.J.; ISLAM, M.K.; JAHAN, I.A.; HOSSAIN, H. 2016. Antiinflammatory and antioxidant activity of Acalypha hispida leaf and analysis of its major bioactive polyphenols by HPLC. Advanced Pharmaceutical Bulletin. 6(2):275-283. https://dx.doi.org/10.15171/apb.2016.039

30. SOTO VÁSQUEZ, M.R. 2015. Estudio fitoquímico y cuantificación de flavonoides totales de las hojas de Piper peltatum L. y Piper aduncum L. procedentes de la región Amazonas. In Crescendo. Institucional. 6(1):105-116.
31. TERÁN PORTELLES, E.C.; CUÉLLAR CUÉLLAR, A.; SALAS OLIVET, E.; PARDO ANDREU, G.L. 2019. Toxicidad aguda del extracto hidroalcohólico de Croton wagneri Müll. Arg. (moshquera) y su efecto irritante sobre la mucosa bucal. Revista Cubana de Plantas Medicinales. 24(2):e791.

32. TREJO I., C. 2004. Anticoagulantes: Farmacología, mecanismos de acción y usos clínicos. Cuadernos de cirugía. 18(1):83-90.

33. WARDOYO, E.R.P.; RACHMAWATI, U.H.; KURNIATUHADI, R. 2021. Phytochemical analysis and antifungi activity of methanol extract of Acalypha bispida Burm. F. flower against to Candida albicans (Y116). Journal of Physics: Conference Series. 1940(2021):012056. http://dx.doi.org/10.1088/1742-6596/1940/1/012056

34. WARKENTIN, T.E.; LEVINE, M.N.; HIRSH, J.; HORSEWOOD, P.; ROBERTS, R.S.; GENT, M.; KELTON, J.G. 1995. Heparin-induced thrombocytopenia in patients treated with low-molecular-weight heparin or unfractionated heparin. The New England Journal of Medicine. 332(20):1330-1336.

https://doi.org/10.1056/nejm199505183322003

35. ZAMORA-GONZÁLEZ, Y. 2012. Pruebas del coagulograma y componentes de la hemostasia. Utilidad para diagnosticar las diátesis hemorrágicas. Revista Cubana de Hematología, Inmunología y Hemoterapia. 28(2):141-150. 